\title{
Parietal Lobe Lesions Disrupt Saccadic Remapping of Inhibitory Location Tagging
}

\author{
Ayelet Sapir ${ }^{1}$, Amy Hayes ${ }^{2}$, Avishai Henik ${ }^{1}$, Shai Danziger ${ }^{1}$, \\ and Robert Rafal ${ }^{2}$
}

\begin{abstract}
Maintaining a coherent percept of the visual scene while eye position continuously changes requires that saccades be accompanied by remapping of the visual environment. We studied saccadic remapping in patients with unilateral lesions in the intraparietal sulcus and healthy controls, using inhibition of return (IOR) — an inhibitory tag that enables efficient visual search. In healthy controls, IOR was found at both retinal and environmental locations of the cue, indicating that the inhibitory tag had been remapped into environmental coor-
\end{abstract}

\section{INTRODUCTION}

How are representations of a visual scene integrated across eye movements to provide a coherent percept of the environment over time? A static object projects onto a location of the retina and this information is relayed to the visual cortex. When our eyes move, the object's location on the retina changes, but it is nevertheless perceived as a static object that occupies the same environmental location. For this to happen, the visual system must take into account the parameters of the eye movement when integrating the representations preceding and following the eye movement. Neurophysiological (Colby, Duhamel, \& Goldberg, 1996; Duhamel, Colby, \& Goldberg, 1992) and neuropsychological (Heide, Blankenburg, Zimmermann, \& Kompf, 1995; Duhamel, Goldberg, FitzGibbon, Sirigu, \& Grafman, 1992) studies have implicated the parietal lobe in the remapping of the visual field following eye movements. Here, we provide novel evidence that the region of the intraparietal sulcus is also involved in remapping an inhibitory tag that enables efficient visual search.

\section{Environmental Coding and Saccade Remapping}

Visually responsive neurons in the lateral intraparietal (LIP) cortex have retinotopic receptive fields (e.g., Colby et al., 1996) but also use information about intended eye

\footnotetext{
${ }^{1}$ Ben-Gurion University of the Negev, ${ }^{2}$ University of Wales
}

dinates. In contrast, right parietal patients demonstrated IOR only at the retinal location of the cue, indicating that the intraparietal sulcus is involved in remapping of the environment after eye movements to afford a stable, environmentally based reference frame. Note that patients did not show environmental IOR in either visual field. These results also suggest that this region may be the neural substrate for encoding inhibitory spatial tags in an environmentally based reference frame.

movement to predict the retinal result of the movement and update the representation of the visual field (Duhamel, Colby, et al., 1992). Thus, most LIP neurons discharge when a saccade brings a recently flashed stimulus into the retinal receptive field even if the stimulus disappeared before the saccade brought it into the receptive field. Hence, these neurons can update the retinal coordinates of remembered stimuli to generate a continuous representation of visual space (Duhamel, Colby, et al., 1992).

Duhamel, Goldberg, et al. (1992) provided clinical evidence for the involvement of the parietal lobe in the remapping of the visual environment across saccades. They tested a patient with a hemorrhage in the right frontoparietal cortex in a double-step saccade paradigm. In their experiment, two brief flashes of light were presented one after the other, and the patient was instructed to move her eyes to the flashes in the order of their appearance. In the critical condition, the two targets disappeared before the patient moved her eyes. Here, an accurate first saccade required the target's retinal coordinates while an accurate second saccade required the patient to "calculate" the second target's location relative to the new eye position. In other words, an accurate second saccade required remapping of the visual field to update its location in environmental coordinates. It was found that contralesional and ipsilesional first saccades were accurate, but that the patient was unable to make the second saccade accurately if the first saccade was toward the contralesional field. On these findings, they suggested that each 
hemisphere is responsible for programming eye movements to the contralateral field and generating the signal used for remapping the visual environment.

Subsequently, Heide et al. (1995) tested groups of patients with various cortical lesions using the same paradigm and found that posterior parietal patients were unable to make an accurate second saccade following a contralesional first saccade. Parietal lobe (and in particular the LIP) activation during remapping was also found in a functional magnetic imaging study using a doublestep saccade paradigm (Tobler et al., 2001).

\section{Environmental Coding and Inhibitory Tagging}

In the current study, we examined the effect of parietal lesions on the remapping of an inhibitory tag called inhibition of return (IOR; Posner \& Cohen, 1984). IOR is a phenomenon characterized by slow responding to targets that appear at recently cued locations. In a typical IOR paradigm, participants are presented with three boxes on a computer screen, one in the center and the other two equidistant from it in the left and right visual fields. After fixating the center box, one of the two peripheral boxes flashes briefly (the cue). This cue is followed by a target that appears, with equal probability, in either the cued or the opposite box. Participants are asked to press a key as soon as they detect the target. When the interval is longer than approximately $300 \mathrm{msec}$, participants respond slower to a target appearing at the cued than at the uncued location. Posner \& Cohen (1984) speculated that this inhibitory tag enables us to search the environment more efficiently (Muller \& von Muhlenen, 2000; Takeda \& Yagi, 2000; Klein, 1988; Klein \& MacInnes, 1999), giving priority to novel locations in the visual field by inhibiting locations already inspected.

If this inhibitory tag is to be useful in guiding efficient visual search, it must persist in time across successive eye movements and be mapped in environmental coordinates. Consistent with this assumption, IOR persists for at least several seconds (Berlucchi, Chelazzi, \& Tassinari, 2000) and is mapped in environmental rather than retinal coordinates (Maylor \& Hockey, 1985; Posner \& Cohen, 1984). That is, if subjects move their eyes after the cue but before the target, IOR appears at the environmental location of the cue.

Several studies have now shown that IOR is generated through retinotectal pathways to the superior colliculus (Sapir, Soroker, Berger, \& Henik, 1999; Danziger, Fendrich, \& Rafal, 1997; Rafal, Calabresi, Brennan, \& Sciolto, 1989; Rafal, Posner, Friedman, Inhoff, \& Bernstein, 1988). The superior colliculus is a midbrain structure that is part of a phylogenetically older visual system for reflexive eye movements. Because the superior colliculus represents eye movement signals in retinal coordinates (or vector code, Schiller, 1998), it has been suggested that the inhibitory tag it generates must be transmitted to higher cortical structures that are mapped in environmental coordinates (Tipper et al., 1997). Klein (2000) has suggested the parietal lobe as a structure that maintains the environmental coordinates of locations tagged by the superior colliculus.

In the current study, we probed for IOR in both retinal and environmental coordinates in patients with chronic, unilateral lesions in the region of the intraparietal sulcus (Figure 1 shows a reconstruction of the lesions; Table 1 provides patient descriptions) and in normal controls. The experimental paradigm is depicted in Figure 2. The display consisted of four boxes that formed an imaginary square. One box was above and one below a plus sign in the center of the screen that subjects fixated at the beginning of each trial. The other two boxes were, in separate blocks, either to the left or right (i.e., either the ipsilesional or the contralesional field of the patients). A participant's task was to press a key when a target appeared inside one of the four boxes. Each trial began with a precue - a flashing of one of the boxes located above or below fixation-that was not informative about the location of the forthcoming target. There were two conditions in the experiment: in one, fixation was maintained throughout the block. Here, the environmental and retinal locations of the cue were identical and IOR was expected to occur at the cued location. In the other, participants moved their eyes to a marker between the two peripheral boxes sometime between cue and target presentation. Here the target could occur (1) at the

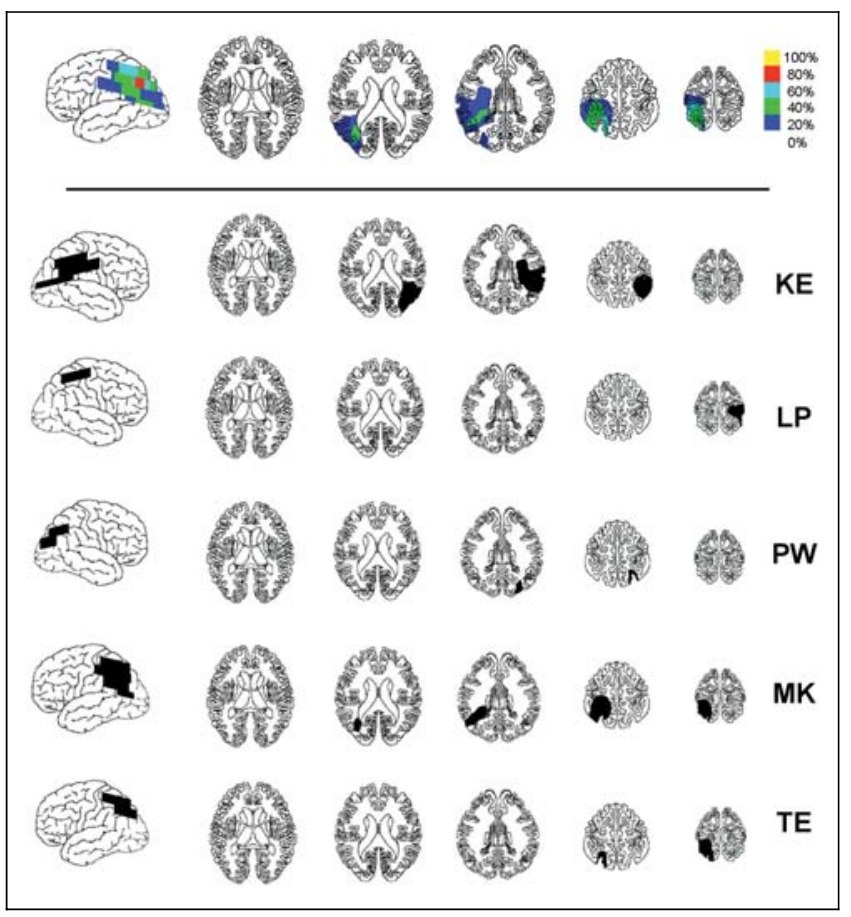

Figure 1. Reconstruction of brain scans of each patient and a group average. The shading indicates the number of patients with a lesion in the area indicated. The region of maximal overlap is the superior part of the intraparietal sulcus. 
Table 1. Patient Clinical Information

\begin{tabular}{|c|c|c|c|c|c|c|}
\hline \multirow[b]{2}{*}{ Patient } & \multirow[b]{2}{*}{ Sex } & \multirow[b]{2}{*}{ Age } & \multicolumn{4}{|c|}{ Lesion } \\
\hline & & & Side & Chronicity (years) & Etiology & Volume (cc) \\
\hline \multirow[t]{2}{*}{ K.E. } & Female & 48 & Right & 23 & Glioma & 46 \\
\hline & & & & & Resection & \\
\hline L.P. & Male & 72 & Right & 8 & Stroke & 6 \\
\hline P.W. & Female & 59 & Right & 1 & Stroke & 5 \\
\hline M.K. & Male & 51 & Left & 30 & Shrapnel & 33 \\
\hline T.E. & Female & 46 & Left & 3 & Stroke & 11 \\
\hline
\end{tabular}

environmental location of the precue (the box that was flashed, which was above or below fixation at the time of the precue, but was lateralized to either the left or right field when the target appeared there), (2) at the retinal location of the precue (i.e., projected to the same point on the retina that was stimulated by the precue, and was at the midline, above or below fixation, when the target appeared), or (3) at one of the two uncued locations (either lateralized or at the midline). Environmentally based IOR was measured as a slower detection reaction time (RT) when the target appeared in the box that had been cued (that was lateral to the second fixation) compared to the uncued lateralized box. Retinally based IOR was measured as a slower detection RT when the target appeared in the midline location (retinally cued location) compared to the other midline location (retinally uncued location).

We expected that normal controls would manifest IOR at the environmental location of the cue (Maylor \& Hockey, 1985; Posner \& Cohen, 1984). We hypothesized that this environmental coding of IOR would depend upon remapping of the visual field in the oculomotor parietal cortex and predicted that patients with parietal damage would be unable to remap the environment after moving their eyes. Thus, patients were predicted to manifest IOR in the retinal rather than the environmental location of the cue.

\section{RESULTS}

The fixation condition was entered into the experiment to verify static IOR. In this condition, the joint analysis of both patients and controls revealed a significant cue validity effect, $F(1,15)=9.73, p<.01$, with valid trials slower then invalid trials (IOR), while the Cue Validity $x$ Group interaction was not significant, $F<1$, indicating that IOR in the static condition was observed in both groups. Note that in this condition, only trials in which the target appeared above or below fixation were included in the analysis.

In the eye movement condition, separate analyses were conducted for controls and patients, because controls did not have "contralesional" or "ipsilesional" visual fields. Median RTs for each experimental condition for each participant were subjected to a four-way repeated measures analysis of variance, with the factors Block (saccade to left vs. saccade to right field), Target Location (environmental, i.e., lateralized at the time of the target vs. retinal, i.e., midline at the time of the target), Cue Validity (target at cued vs. uncued locations), and stimulus onset asynchrony (SOA, between the cue and target). In the patient group, we also tested the effect of lesion side.

As shown in Table 2, for both groups there was no effect of Block (left vs. right saccade in the controls, and contralesional vs. ipsilesional saccade in the patients), nor did Block interact with other factors. Figure 3 shows the mean RTs for each condition collapsed across Block.

\section{Controls}

RTs were shorter at the longer cue-target longer cuetarget SOA, $F(1,11)=21.59, p<.001$, and were faster for

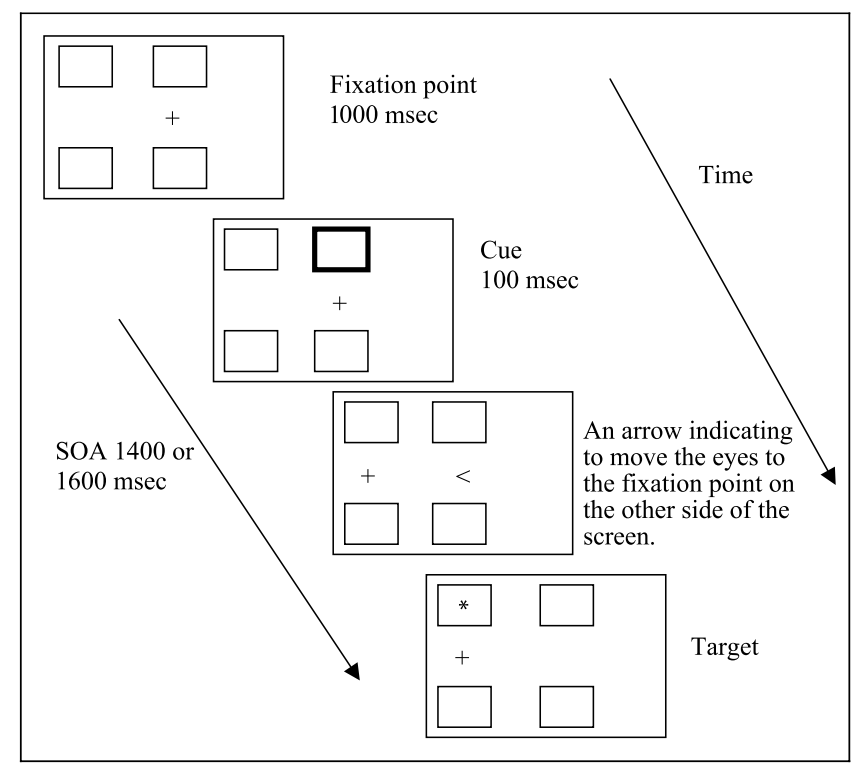

Figure 2. Experimental procedure, retinally valid trial (see text). 
Table 2. Mean RT (in msec) to Detect Target in Each Condition for Controls and Patients

\begin{tabular}{lcccc}
\hline & $\begin{array}{c}\text { Right Visual Field } \\
\text { (Environmental } \\
\text { Controls }\end{array}$ & $\begin{array}{c}\text { Midline (Retinal } \\
\text { Cued vs. Uncued) }\end{array}$ & $\begin{array}{c}\text { Left Visual Field } \\
\text { (Environmental } \\
\text { Cued vs. Uncued) }\end{array}$ & $\begin{array}{c}\text { Midline (Retinal } \\
\text { Cued vs. Uncued) }\end{array}$ \\
\hline Cued & 470 & 445 & 460 & 433 \\
Uncued & 458 & 431 & 436 & 427 \\
IOR & 12 & 14 & 24 & 6
\end{tabular}

\begin{tabular}{|c|c|c|c|c|}
\hline Patients & $\begin{array}{l}\text { Contralesional Visual } \\
\text { Field (Environmental } \\
\text { Cued vs. Uncued) }\end{array}$ & $\begin{array}{l}\text { Midline (Retinal } \\
\text { Cued vs. Uncued) }\end{array}$ & $\begin{array}{l}\text { Ipsilesional Visual } \\
\text { Field (Environmental } \\
\text { Cued vs. Uncued) }\end{array}$ & $\begin{array}{l}\text { Midline (Retinal } \\
\text { Cued vs. Uncued) }\end{array}$ \\
\hline Cued & 517 & 530 & 539 & 530 \\
\hline Uncued & 520 & 494 & 535 & 504 \\
\hline IOR & -3 & 36 & 4 & 26 \\
\hline
\end{tabular}

targets on the midline (above and below fixation at the time of target appearance) than for lateralized targets (that had been above or below fixation at the time of the cue), $F(1,11)=15.44, p<.01$. Most importantly, RTs were longer for targets appearing at cued locations (i.e., IOR), $F(1,11)=5.49, p<.05$. IOR was manifest in both environmental (18 $\mathrm{msec})$ and retinal $(10 \mathrm{msec})$ reference frames. The larger IOR for environmental than retinal locations was not statistically reliable, $F<1$.

\section{Patients}

RTs were shorter at the longer cue-target SOAs, $F(1,4)=$ $14.26, p<.05$. The key finding was a two-way interaction between Target Location and Cue Validity, $F(1,4)=$ $30.76, p<.01$, as IOR was present in the retinal (32 msec) but not the environmental (1 $\mathrm{msec})$ reference frame (see Figure 3). Lesion side was not significant but we found a three-way interaction of Lesion Side $\times$ Target Location $\times$ Cue Validity, $F(1,4)=12.38, p<.05$, suggesting that the difference between retinal and environmental IOR is present in right but not in left parietal patients (see Table 3 for IOR of individual patients). Given the small number of patients, this difference should be replicated in a different study.

\section{DISCUSSION}

The results in healthy controls confirmed earlier works showing that a peripheral visual cue generates both environmentally and retinally based IOR (Posner \& Cohen, 1984, but see Maylor \& Hockey, 1985). In contrast, patients with lesions of the oculomotor parietal cortex in the region of the intraparietal sulcus showed IOR only at the retinal location of the cue. This finding suggests a critical role for parietal oculomotor structures in establishing the environmentally based coordinates of inhibitory tags generated through retinotectal pathways.

\section{Neural Circuitry of Inhibitory Tagging}

Converging evidence has implicated the retinotectal pathway in the generation of IOR. IOR is disrupted in patients with midbrain lesions due to degeneration from progressive supranuclear palsy (Posner, Rafal, Choate, \& Vaughn, 1985) or to unilateral vascular damage (Sapir et al., 1999). In addition, IOR is generated even when visual processing in the geniculostriate pathway is not available. For example, it is generated in newborn infants in whom the colliculus is functional but striate cortex is not (Simion, Valenza, Umilta, \& Dalla Barba, 1995). Moreover, IOR has been demonstrated in the hemianopic field of a patient with striate cortex damage, even in the absence of awareness of the stimulus that evokes it (Danziger et al., 1997). Recently, Dorris, Klein, Everling, and Munoz (2002) recorded from the superior colliculus while the monkey performed an IOR task and found a reduction in the sensory responses in the superior colliculus but the superior colliculus itself was

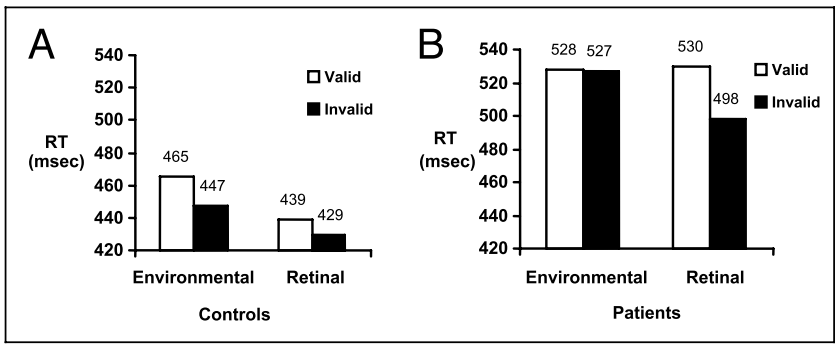

Figure 3. RTs in milliseconds for controls (A) and patients (B) in the move condition. 
Table 3. IOR in msec (Valid Minus Invalid) in Each Condition for Each Patient

\begin{tabular}{|c|c|c|c|c|c|c|}
\hline \multirow[b]{2}{*}{ Patient } & \multirow[b]{2}{*}{ Lesion Side } & \multicolumn{2}{|c|}{ Environmental IOR } & \multicolumn{2}{|c|}{ Retinal IOR } & \multirow[b]{2}{*}{ Fixed IOR } \\
\hline & & Ipsilesional & Contralesional & Ipsilesional & Contralesional & \\
\hline K.E. & Right & 10 & -32 & 33 & 9 & -23 \\
\hline L.P. & Right & 1 & -19 & 16 & 54 & 21 \\
\hline P.W. & Right & -24 & -33 & 37 & -11 & 17 \\
\hline M.K. & Left & 13 & 34 & 33 & 42 & 47 \\
\hline T.E. & Left & 21 & 33 & 14 & 88 & 67 \\
\hline
\end{tabular}

not inhibited but was actually more active when the target appeared at the previously cued location.

The hemianopia study (Danziger et al., 1997) used a paradigm similar to that used here. Unseen cue was presented above or below the horizontal meridian in the blind field. Then, a saccade was made such that a subsequent target appeared above or below fixation at the cued or the uncued location. Two hemianopic patients were studied. One patient showed IOR in both intact and blind fields, demonstrating that environmentally coded IOR can be generated through extrageniculostriate pathways. However, the second patient showed IOR only in the intact field. This patient had an additional lesion in the central thalamus, a nucleus connected to the cortical oculomotor areas that has been shown to be involved in saccadic remapping of the visual environment (Gaymard, Rivaud, \& Pierrot-Deseilligny, 1994). We speculated that the failure to observe environmental IOR was due to a failure of remapping of the inhibitory tag after the saccade was made.

Tipper et al. (1997) have suggested that although IOR may be generated by the colliculus, the inhibitory tag must be transmitted to some cortical representation if it is to guide visual search in everyday situations, where objects and the eyes are continuously moving. They provided support for a cortical contribution to IOR from a study with split-brain patients. In that study after a box (object) was cued, it was moved, together with an uncued object, before the target appeared in either the cued or uncued box. In normal individuals, IOR was found in the cued object although it had moved to a different retinal location. In two split-brain patients, object-based IOR was present only if the cued object moved within the same hemifield. The involvement of the corpus callosum in transferring the tag from one visual field to another suggests a cortical substrate for representing the inhibitory tag in objectbased coordinates.

In the current study, the failure to remap IOR in environmental coordinates in patients with parietal lesions could, as suggested above, reflect the importance of the oculomotor parietal cortex for remapping the visual environment when saccades are made. This region could also be the cortical substrate where the inhibitory tag is encoded in environmental coordinates. Both possibilities are considered next.

\section{Oculomotor Parietal Cortex and Remapping of the Visual Environment}

The current results, using IOR as a marker of visual remapping, are consistent with previous work using the double-step saccade paradigm (Heide et al., 1995; Duhamel, Goldberg et al., 1992) that have implicated the oculomotor parietal cortex in remapping of the visual environment. One difference between these studies and ours is that, in the current study, remapping was absent whether saccades were made to either ipsilesional or contralesional fields. Studies using the double-step saccade paradigm have shown a failure of remapping specific to the contralesional field.

Why are the deficits in environmental coding of IOR reported here bilateral? As mentioned, it may be that the deficits reported here do not simply reflect a failure of saccadic remapping, but rather that the parietal cortex is actually the neural substrate for encoding the inhibitory tag in an environmental reference frame. When patients made saccades toward the contralesional field, the lesion prevented remapping of the visual field resulting in retinal rather than environmental coding. In contrast, when patients made saccades toward the ipsilesional field remapping was presumably successful. Note, however, that in this case, a target presented at the environmental location of the cue was in the contralesional field and, thus, projected to the damaged hemisphere. If the remapped coordinates provided by the intact hemisphere cannot be encoded in the lesioned hemisphere processing the target, then the inhibitory tag will remain in retinal coordinates as was indeed found in our experiment. This suggests that the intraparietal region is not only involved in remapping the visual field during saccades, but also in integrating this remapped information in a cortical representation of the inhibitory tag.

In summary, we report that chronic unilateral lesions centered in the region of the intraparietal sulcus result in 
a failure of environmental inhibitory location tagging in both the contralesional and ipsilesional fields. These observations are consistent with both neurophysiological and neuropsychological studies that implicate the parietal cortex in remapping of the visual field to afford a stable, environmentally based reference frame. They also suggest that this region may be the neural substrate for encoding inhibitory spatial tags in an environmentally based reference frame.

\section{METHODS}

\section{Participants Description}

Five patients and 12 controls participated after giving informed consent. The study was approved by the ethics committees of the University of California, Davis, the Martinez VA hospital, the University of Wales, and the Northwest Wales NHS Trust. All patients were chronic ( $>1$ year), with small (lesion volumes ranging between 5 and $46 \mathrm{cc}$; mean $20 \mathrm{cc}$ ), unilateral lesions in the region of the intraparietal sulcus (Table 1). For four patients, the lesion involved the medial bank, the lateral bank, or both of the intraparietal sulci. In the fifth patient, a lesion restricted to the superior parietal lobule undercuts the lateral bank of the intraparietal sulci. There were two men and three women ranging in age between 48 and 73 (mean 55) years old. None of the patients had neurological signs on clinical examination. CT scans were available for all the patients and magnetic resonance imaging scans were obtained for two of them. From these scans, the lesions were manually transcribed onto standard templates for reconstruction (Figure 1). The healthy controls included 6 men and 6 women ranging in age between 37 and 82 (mean 62) years old.

\section{Experimental Procedure}

The display was controlled by a Macintosh computer that also recorded reaction times (RT) from responses made on the button of a joystick placed in front of the participant. The participants sat in a dark room, facing the computer screen at eye level at a distance of $57 \mathrm{~cm}$. The constant display consisted of four unfilled black boxes on a white background at the corners of an imaginary square. Each box was $32 \times 32 \mathrm{~mm}$, with a line thickness of $1 \mathrm{~mm}$. One box was centered $54 \mathrm{~mm}$ above the fixation point and another was centered $54 \mathrm{~mm}$ below it. The other two boxes were equidistant and in line with the boxes in the center of the display. In half the blocks, they were to the left of fixation, and in the other half, to the right. Thus, for the move condition (see below), performance for contralesional and ipsilesional fields was tested in separate blocks.

Each trial began with the appearance of a plus sign, that participants were instructed to fixate, in the center of the screen midway between two of the boxes located equidistant above and below the fixation point. A precue was presented $1000 \mathrm{msec}$ later by flashing one of these boxes for $100 \mathrm{msec}$. This precue was not informative about the location of the forthcoming target. The precue was generated by changing one of the squares to a thickness of $6 \mathrm{~mm}$. Either 1400 or $1600 \mathrm{msec}$ after the onset of the precue the target (an asterisk of the same size as the fixation cross) appeared, randomly and with equal probability, in one of the four boxes, and the participants made a manual key press response. The target remained visible until response. If no response was made within $1000 \mathrm{msec}$, the trial was terminated. The intertrial interval was $1500 \mathrm{msec}$.

Two conditions were mixed in each block with equal probability: in one, fixation was maintained throughout the trial; in the other, an eye movement was required after the cue and before the target appeared. In the fixation condition, the fixation point remained visible throughout the trial. In the eye movement condition, 500 msec after the onset of the cue, the fixation cross was replaced by an arrowhead, subtending $12 \mathrm{~mm}$ vertically and $8 \mathrm{~mm}$ horizontally, pointing toward a small white box located between the boxes in the opposite visual field. Participants were asked to make an eye movement to fixate this box, and after an eye movement was made, the target appeared. Thus, the target could appear at the environmental location of the precue (the same box that was flashed), at the retinal location of the precue (i.e., projected to the same point on the retina that was stimulated by the precue) or at one of the other two uncued locations.

The experimenter sat in front of the participant and cancelled the trial if an eye movement was not made or the new location was not fixated before the target appeared. The participants were tested in two separated blocks, one that required eye movements to the left and one that required eye movements to the right. Within each session 12 practice trials for each condition (fixation and move) and an additional 16 mixed trials preceded 256 test trials.

\section{Acknowledgments}

This research was supported by an NIMH grant, RO1 MH41544, to R. Rafal.

Reprint requests should be sent to Ayelet Sapir, Radiology Department, Washington University School of Medicine, Box 8225, 4525 Scott Avenue, St. Louis, MO, 63110, USA, or via e-mail: ayelet@nil.wustl.edu.

\section{REFERENCES}

Berlucchi, G., Chelazzi, L., \& Tassinari, G. (2000). Volitional covert orienting to a peripheral cue does not suppress cue-induced inhibition of return. Journal of Cognitive Neuroscience, 12, 648-663. 
Colby, C. L., Duhamel, J. R., \& Goldberg, M. E. (1996). Visual, presaccadic and cognitive activation of single neurons in monkey lateral intraparietal area. Journal of Neurophysiology, 76, 2841-2852.

Danziger, S., Fendrich, R., \& Rafal, R. D. (1997). Inhibitory tagging of locations in the blind field of hemianopic patients. Consciousness and Cognition, 6, 291-307.

Dorris, M. C., Klein, R. M., Everling, S., \& Munoz, D. P. (2002). Contribution of the primate superior colliculus to inhibition of return. Journal of Cognitive Neuroscience, 14 , 1256-1263.

Duhamel, J. R., Colby, C. L., \& Goldberg, M. E. (1992). The updating of the representation of visual space in parietal cortex by intended eye movements. Science, 255, 90-92.

Duhamel, J. R., Goldberg, M. E., FitzGibbon, E. J., Sirigu, A., \& Grafman, J. (1992). Saccadic dysmetria in a patient with a right frontoparietal lesion: The importance of corollary discharge for accurate spatial behavior. Brain, 115, 1387-1402.

Gaymard, B., Rivaud, S., \& Pierrot-Deseilligny, C. (1994). Impairment of extraretinal eye position signals after central thalamic lesions in humans. Experimental Brain Research, 102, 1-9.

Heide, W., Blankenburg, M., Zimmermann, E., \& Kompf, D. (1995). Cortical control of double-step saccades: Implications for spatial orienting. Annals of Neurology, 38, 739-748.

Klein, R. (1988). Inhibitory tagging system facilitates visual search. Nature, 334, 430-431.

Klein, R. M. (2000). Inhibition of return. Trends in Cognitive Sciences, 4, 138-147.

Klein, R. M., \& MacInnes, W. J. (1999). Inhibition of return is a foraging facilitator in visual search. Psychological Science, 10, 346-352.

Maylor, E. A., \& Hockey, R. (1985). Inhibitory component of externally controlled covert orienting in visual space. Journal of Experimental Psychology: Human Perception and Performance, 11, 777-787.

Muller, H. J., \& von Muhlenen, A. (2000). Probing distractor inhibition in visual search: Inhibition of return. Journal of Experimental Psychology: Human Perception and Performance, 26, 1591-1605.

Posner, M. I., \& Cohen, Y. (1984). Components of visual orienting. In H. Bouma \& D. Bouwhuis (Eds.), Attention and Performance $X$ (pp. 531-556). London: Erlbaum.

Posner, M. I., Rafal, R. D., Choate, L., \& Vaughn, J. (1985). Inhibition of return: Neural basis and function. Cognitive Neuropsychology, 2, 211-228.

Rafal, R., Calabresi, P., Brennan, C., \& Sciolto, T. (1989). Saccade preparation inhibits reorienting to recently attended locations. Journal of Experimental Psychology: Human Perception and Performance, 15, 673-685.

Rafal, R. D., Posner, M. I., Friedman, J. H., Inhoff, A. W., \& Bernstein, E. (1988). Orienting of visual attention in progressive supranuclear palsy. Brain, 111, 267-280.

Sapir, A., Soroker, N., Berger, A., \& Henik, A. (1999). Inhibition of return in spatial attention: Direct evidence for collicular generation. Nature Neuroscience, 2, 1053-1054.

Schiller, P. H. (1998). The neural control of visually guided eye movements. In J. E. Richards (Ed.), Cognitive neuroscience of attention: A developmental perspective (pp. 3-50). Mahwah, NJ: Erlbaum.

Simion, F., Valenza, E., Umilta, C., \& Dalla Barba, B. (1995). Inhibition of return in newborns is temporo-nasal asymmetrical. Infant Behavior and Development, 18, 189-194.

Takeda, Y., \& Yagi, A. (2000). Inhibitory tagging in visual search can be found if search stimuli remain visible. Perception and Psychophysics, 62, 927-934.

Tipper, S., Rafal, R., Reuter-Lorenz, P. A., Starrveldt, Y., Ro, T., Egly, R., Danzinger, S., \& Weaver, B. (1997). Object-based facilitation and inhibition from visual orienting in the human split-brain. Journal of Experimental Psychology: Human Perception and Performance, 23, 1522-1532.

Tobler, P. N., Felblinger, J., Burki, M., Nirkko, A. C., Ozdoba, C., \& Muri, R. M. (2001). Functional organization of the saccadic reference system processing extraretinal signals in humans. Vision Research, 41, 1351-1358. 